\title{
Atomwaffen - der lange Weg der Schweiz
}

\author{
Im Vorfeld des Weltkongresses der International Physicians for the Prevention of \\ Nuclear War IPPNW trafen Schweizer Vertreter dieser Organisation Peter Maurer, \\ Staatssekretär des EDA, zum Gespräch. Dabei wurden neben aktuellen Fragen zur \\ globalen nuklearen Abrüstung auch historische Aspekte des "Schweizer Wegs" in \\ Sachen Atomwaffen diskutiert. Der Staatssekretär zeigte sich an einer Zusammen- \\ arbeit mit IPPNW interessiert.
}

Erhard Taverna

erhard.taverna@saez.ch

\begin{abstract}
Alle fünf Jahre diskutieren die 189 Unterzeichnerstaaten des Atomwaffensperrvertrags von 1970 (Nonproliferation Treaty, NPT) über Abrüstung und Verzicht auf Nuklearwaffen. Mit der achten dieser Überprüfungskonferenzen (Review Conference, NPT) im Mai 2010 am Hauptsitz der UNO waren besonders viele Hoffnungen verknüpft. Die Schweizer Regierung bekräftigte dabei erstmals ihre klare Unterstützung einer Nuklearwaffenkonvention (Nuclear Weapon Convention, NWC). Im Gegensatz zum Atomwaffensperrvertrag, der in Artikel VI lediglich das Ziel der globalen nuklearen Abrüstung erwähnt, geht die Nuklearwaffenkonvention weiter und listet die einzelnen Schritte auf, die zu diesem Ziel führen. Als erster Schritt wurde zum Beispiel die Rückstufung der immer noch hohen Alarmbereitschaft vieler ballistischer Atomwaffen der Nuklearmächte vorgeschlagen. Ein Ansatz, der viel weiter geht als der bisherige Sperrvertrag. Die Aussen-
\end{abstract}

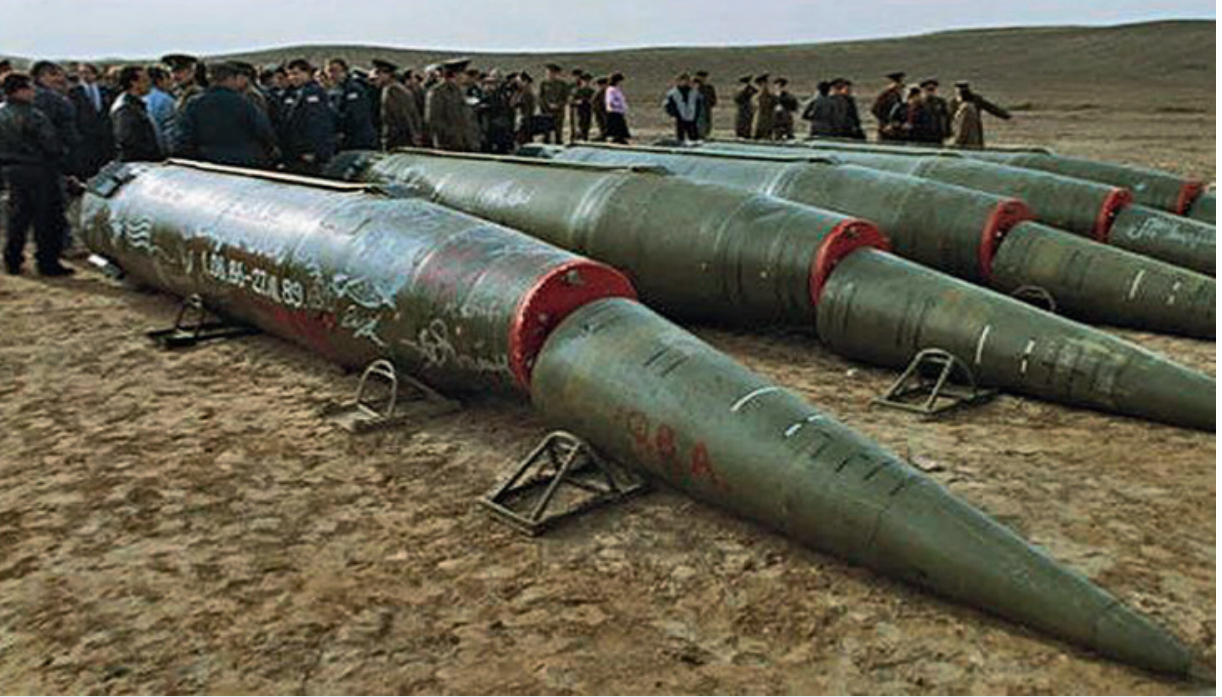

Ein Schritt in die richtige Richtung: Demontage nuklearer Waffen. ministerin Calmy-Rey bezeichnete in New York Atomwaffen als «nicht einsetzbar, unmoralisch und illegal». Sie setzte sich vor der Versammlung für eine völkerrechtliche Ächtung ein, mit dem Ziel einer internationalen Verbotskonvention, wie sie für chemische und biologische Waffen besteht.

Das war nicht immer so. Bis 1969 unternahm die Schweiz grosse Anstrengungen, um im Alleingang Atomwaffen zu entwickeln. Das Reaktorunglück von Lucens und der Mirageskandal beendeten den Traum der nuklearen Eigenständigkeit. Zwei Abstimmungen, 1962 und 1963, hatten eine eindeutige Volksmehrheit zugunsten der Bewaffnungspläne des Bundesrates ergeben. Dennoch unterzeichnete die Schweiz 1963 ein partielles Atomteststoppabkommen und 1969 den Atomwaffensperrvertrag, der allerdings wegen der Opposition im Ständerat erst 1976 genehmigt wurde. Die Sicherheitspolitik wechselte von der aktiven Strategie zur passiven Verteidigung der Bevölkerung durch Schutzräume. 1988 wurde die Kernwaffenoption endgültig fallengelassen, 1995 stimmte die Schweiz der unbefristeten Verlängerung des Atomsperrvertrags zu und damit auch einem effizienten Überprüfungssystem durch die UNO.

\section{Im Bundeshaus}

Der neue Staatssekretär Peter Maurer im Departement für auswärtige Angelegenheiten EDA, bis vor kurzem unser Vertreter am UNO-Sitz in New York, ist soeben aus den USA zurückgekehrt. Flankiert von seinem Leiter des politischen Sekretariats Andreas Friedrich, zuständig für internationale Sicherheitspolitik und Rüstungskontrolle, schildert er seine Eindrücke und erläutert die Ziele der Bundesrätin. Sein Departement richte die Agenda nach dem Realisierbaren, man müsse abwarten, wie die Welt nach der Konferenz aussehe. Die Iran-Frage und innenpolitisches Taktieren hätten für Präsident Obama erste Priorität. Bis Ende der 90er Jahre sei der nukleare Schutzschild der USA für die Schweiz eine nicht weiter zu hinterfragende Tatsache gewesen. 
Diese Positionen hätten sich langsam geändert. Zwar seien die ehemaligen Ostblockländer an einer atomwaffenfreien Zone in Europa nicht interessiert, aber mit Österreich könne man reden. Zusammen mit anderen atomaren Habenichtsen wie Neuseeland und Irland nütze die Schweiz ihre guten Verbindungen zum Internationalen Komitee vom Roten Kreuz IKRK und innerhalb der UNO.

Ein Schwerpunkt liege auf der angestrebten weltweiten Verbannung von Atomwaffen. Die Schweiz stellt sich als Plattform für die wichtigsten Akteure zur Verfügung. Eine Studie im Auftrag des EDA liefert dazu interessante Argumente für eine Verbotskonvention. «Delegitimizing Nuclear Weapons, Examining the validity of nuclear deterrence» ist der Titel dieser Untersuchung des Center for Nonproliferation Studies CNS am Monterey Institute of International Studies MIIS in Kalifornien [1]. Die renommierte Privatuniver-
Menschen mobilisiert hat als heute. Denn seither wurden die Waffen weiter verbreitet. Labile Staaten und diktatorische Regierungen treiben ungehindert den Ausbau ihrer Arsenale voran. Die internationale Kontrollbehörde IAEA (Internationale Atomenergie Agentur) in Wien registriert immer wieder neue Versuche krimineller und terroristischer Gruppen, in den Besitz von radioaktivem Material zu gelangen. Der Gedanke, dass zukünftige Konflikte um Land, Energie, Nahrung und Wasser in einer überbevölkerten Welt mit diesen Waffen ausgetragen werden, ist unerträglich. Seit 1945 versucht ein komplexes Vertragswerk, mit mehr oder weniger Erfolg ein Wettrüsten einzudämmen, doch die Gegenwart zeigt, dass dies nicht genügt. Tausende von Nuklearwaffen sind auf einem hohen Bereitschaftsgrad. Kritische Vorfälle der Vergangenheit zeigen, dass auch ein unbeabsichtigter Nuklearkrieg möglich ist.

\section{Kritische Vorfälle der Vergangenheit zeigen, dass auch ein unbeabsichtigter Nuklearkrieg möglich ist}

sität demontiert angebliche Gewissheiten über die Folgen und den Nutzen der atomaren Bewaffnung. Gemäss den Autoren haben nicht die Abwürfe über Hiroshima und Nagasaki Japan zur Kapitulation gezwungen, es war vielmehr die gleichzeitige Kriegserklärung der Sowjetunion. Das Gleichgewicht des Schreckens im kalten Krieg war eine Fiktion, ebenso wie die angebliche militärische Überlegenheit dank Erst- und Zweitschlagkapazitäten. Kein einziger Krieg ist seit 1945 durch das Abschreckungspotential vermieden worden. Atomwaffen können in Zukunft durch andere Statussymbole ersetzt werden. Eine Welt mit weiteren Staaten als Atomwaffenbesitzer ist nicht stabiler als eine Welt mit weniger Atomwaffenstaaten und einer stark verminderten Anzahl dieser Waffen, ganz im Gegenteil. Zahlreiche Beispiele untermauern diese Schlussfolgerungen, zu denen auch die Überlegungen hoher Militärs vieler Länder beigetragen haben. Die Politik des EDA stützt sich unter anderem auf folgende Feststellung aus dieser Untersuchung: «Mobilizing international public and political support, and sustaining it throughout the disarmement process, is perhaps the most fundamental precondition for progress on the path towards a world without nuclear weapons.»

\section{Die Rolle der IPPNW}

Eigentlich ist es paradox, dass zu Zeiten des kalten Krieges die Angst vor einem atomaren Krieg viel mehr
Die Schweiz hat einen weiten Weg zurückgelegt. 2007 und 2008 hat sie mit Ländern wie Chile, Neuseeland, Nigeria, Malaysia und Schweden eine Resolution «Decreasing the Operational Readiness of Nuclear Weapons» in die UNO-Vollversammlung eingebracht. 2009 haben russische und amerikanische Expertenteams sowie Vertreter von Nichtnuklearstaaten in der Schweiz Vorschläge zur Sicherheit atomarer Systeme erarbeitet.

Die Macher der Studie setzen auf Aktionen einer globalen Zivilgesellschaft in einer technisch gut vernetzten Welt: «We need new blood in the debate.» Die Ziele der geplanten Nuklearwaffenkonvention sollen die Öffentlichkeit aufwecken und zur Mitarbeit animieren. Eine gute Chance sehen die Autoren im Potential grosser Frauenbewegungen, religiöser Organisationen und engagierter NGO (Non Governmental Organizations). Besonders hervorgehoben werden $\mathrm{Zu}$ sammenschlüsse von Ärzten, anderen Gesundheitsberufen und Wissenschaftlern aller Richtungen. Kongresse wie derjenige im August 2010 in Basel entsprechen genau diesem Konzept. Aktionen der IPPNW (International Physicians for the Prevention of Nuclear War) wollen informieren und im besten Sinn Unruhe stiften. Der Staatssekretär des EDA hat sein Interesse an einer Zusammenarbeit mit dem IPPNW bekundet [2]. Damit sind alle Kolleginnen und Kollegen zur engagierten Mithilfe aufgerufen. 